\title{
Reducing Noises and Artifacts Simultaneously of Low-Dosed X-Ray Computed Tomography Using Bilateral Filter Weighted by Gaussian Filtered Sinogram
}

\author{
Shaoxiang Hu, ${ }^{1}$ Zhiwu Liao, ${ }^{2}$ and Wufan Chen ${ }^{3}$ \\ ${ }^{1}$ School of Automation Engineering, University of Electronic Science and Technology of China, \\ Chengdu 611731, China \\ ${ }^{2}$ School of Computer Science, Sichuan Normal University, Chengdu 610101, China \\ ${ }^{3}$ Institute of Medical Information and Technology, School of Biomedical Engineering, \\ Southern Medical University, Guangzhou 510515, China \\ Correspondence should be addressed to Zhiwu Liao, liaozhiwu@163.com
}

Received 2 February 2012; Accepted 2 March 2012

Academic Editor: Ming Li

Copyright (C) 2012 Shaoxiang Hu et al. This is an open access article distributed under the Creative Commons Attribution License, which permits unrestricted use, distribution, and reproduction in any medium, provided the original work is properly cited.

Existing sinogram restoration methods cannot handle noises and nonstationary artifacts simultaneously. Although bilateral filter provides an efficient way to preserve image details while denoising, its performance in sinogram restoration for low-dosed X-ray computed tomography (LDCT) is unsatisfied. The main reason for this situation is that the range filter of the bilateral filter measures similarity by sinogram values, which are polluted seriously by noises and nonstationary artifacts of LDCT. In this paper, we propose a simple method to obtain satisfied restoration results for sinogram of LDCT. That is, the range filter weighs the similarity by Gaussian smoothed sinogram. Since smoothed sinogram can reduce the influence of both noises and nonstationary artifacts for similarity measurement greatly, our new method can provide more satisfied denoising results for sinogram restoration of LDCT. Experimental results show that our method has good visual quality and can preserve anatomy details in sinogram restoration even in both noises and nonstationary artifacts.

\section{Introduction}

Radiation exposure and associated risk of cancer for patients receiving CT examination have been an increasing concern in recent years. Thus, minimizing the radiation exposure to patients has been one of the major efforts in modern clinical X-ray CT radiology [1-8]. However, the presentation of strong noises and non-stationary artifacts degrades the quality 
of low-dose CT images dramatically and decreases the accuracy of diagnosis dose. Many strategies have been proposed to reduce the noise, for example, by nonlinear noise filters [8-19] and statistics-based iterative image reconstructions (SIIRs) [20-28].

The SIIRs utilize the statistical information of the measured data to obtain good denoising results but are limited for their excessive computational demands for the large CT image size. Moreover, the mottled noise and non-stationary artifacts in LDCT images cannot be accurately modeled into one specific distribution, which makes it a difficult task to differentiate between noise/artifact and informative anatomical/pathological features [29].

Although the nonlinear filters show effectiveness in reducing noise both in sinogram space and image space, they cannot handle the noise-induced streak artifacts. Since existing methods cannot handle noises and artifacts simultaneously, designing a method to reduce noise and non-stationary artifacts simultaneously becomes an open problem in sinogram restoration of LDCT.

Recently, many new nonlinear filters are presented and show promising denoising performance on space domain [29-44]. Bilateral filter (BF), which integrates range filter (gray level) and domain filter (space) together, is a well-known one $[35,36]$. However, BF cannot obtain satisfied results in sinogram restoration of LDCT because of polluted sinogram values for the range filter. To obtain satisfied denoising results in serious noises, some efforts on image space are proposed [37-41].

Wong suggests that two parameters, $\sigma_{s}^{2}$ and $\sigma_{r}^{2}$, the variances of Gaussian functions in domain and range filters, should be modulated according to local phase coherence of the image pixels [37]. But it blurs edges or leaves uncleaned noises.

Ming and Bahadir improve the performance of BF by multiresolution method [38]. That is, filtering LL subband uses BF while smoothing wavelet subbands uses SURE shrinkage. It also leads to blur edges while denoising.

van Boomgaard and van de Weijer argue that the main reason for unsatisfied denoising results is the polluted center pixel of BF [40]. Thus, the satisfied results can be obtained by replacing polluted center pixel with an estimate of its true gray levels.

Following [40], median bilateral filter (MBF) is proposed in [41]. MBF replaces the center pixel with the median of a $3 \times 3$ window. However, only replacing the center pixel also cannot obtain satisfied denoising results.

Although BF and its improvements can obtain satisfied results in general image denoising, all these methods cannot handle sinogram restoration with noises and non-stationary artifacts simultaneously. We think that the key to handle noises and artifacts simultaneously is how to reduce the influence of both the noises and artifacts of sinogram of LDCT.

In this paper, we propose a new method to reduce the influence of the noises and artifacts of sinogram simultaneously, named bilateral filter weighted by Gaussian filtered sinogram (BFWGFS), which carried on BF on Gaussian smoothed sinogram. Note that, proposed method is different to the method proposed in [40]. The proposed method in [40] only replaces the gray levels of the center point with the median of a $3 \times 3$ square centered at the center point, while our method replaces both the center point and all considering points with their Gaussian smoothed sinogram values.

Since the smoothed sinogram can reduce the influence of both the noises and artifacts of sinogram, the weight of the range filter defined on BFWGFS can measure the similarities more precisely comparing to the original sinogram values in BF. Thus, the proposed method can obtain satisfied results in noises and non-stationary artifacts simultaneously.

In the reminder of this paper, Section 2 will introduce the noise models; then in Section 3, we will discuss the measurement of similarity and discussed the difference between 
the proposed method and method in [40]. Section 4 describes the denoising framework. Section 5 is the experimental results and discussion. Section 6 gives conclusions and finally, the acknowledgment part.

\section{Noise Models}

Based on repeated phantom experiments, low-mA (or low-dose) CT calibrated projection data after logarithm transform were found to follow approximately a Gaussian distribution with an analytical formula between the sample mean and sample variance, that is, the noise is a signal-dependent Gaussian distribution [19].

In this section, we will introduce signal-independent Gaussian noise (SIGN), Poisson noise, and signal-dependent Gaussian noise.

\subsection{Signal-Independent Gaussian Noise (SIGN)}

SIGN is a common noise for the imaging system. Let the original projection data be $\left\{x_{i}\right\}$, $i=1, \ldots, m$, where $i$ is the index of the $i$ th bin. The signal has been corrupted by additive noise $\left\{n_{i}\right\}, i=1, \ldots, m$ and one noisy observation

$$
y_{i}=x_{i}+n_{i}
$$

where $y_{i}, x_{i}, n_{i}$ are observations for the random variables $Y_{i}, X_{i}$, and $N_{i}$ where the upper-case and letters denote the random variables and the lower-cased letters denote the observations for respective variables. $X_{i}$ is normal $N\left(0, \sigma_{X}^{2}\right)$, and $N_{i}$ is normal $N\left(0, \sigma_{N}^{2}\right)$ and independent of the Gaussian random variable $X_{i}$. Thus, $Y_{i}$ is normal $N\left(0, \sigma_{X}^{2}+\sigma_{N}^{2}\right)$.

\subsection{Poisson Model and Signal-Dependent Gaussian Model}

The photon noise is due to the limited number of photons collected by the detector [30]. For a given attenuating path in the imaged subject, $N_{0}(i, \alpha)$ and $N(i, \alpha)$ denote the incident and the penetrated photon numbers, respectively. Here, $i$ denotes the index of detector channel or bin, and $\alpha$ is the index of projection angle. In the presence of noises, the sinogram should be considered as a random process, and the attenuating path is given by

$$
r_{i}=-\ln \left[\frac{N(i, \alpha)}{N_{0}(i, \alpha)}\right]
$$

where $N_{0}(i, \alpha)$ is a constant, and $N(i, \alpha)$ is Poisson distribution with mean $N$.

Thus, we have

$$
N(i, \alpha)=N_{0}(i, \alpha) \exp \left(-r_{i}\right)
$$

Both its mean value and variance are $N$.

Gaussian distributions of ployenergetic systems were assumed based on limited theorem for high-flux levels, and following many repeated experiments in [19], we have

$$
\sigma_{i}^{2}\left(\mu_{i}\right)=f_{i} \exp \left(\frac{\mu_{i}}{\gamma}\right)
$$


where $\mu_{i}$ is the mean, and $\sigma_{i}^{2}$ is the variance of the projection data at detector channel or bin $i, \gamma$ is a scaling parameter, and $f_{i}$ is a parameter adaptive to different detector bins.

The most common conclusion for the relation between Poisson distribution and Gaussian distribution is that the photon count will obey Gaussian distribution for the case with large incident intensity and Poisson distribution with feeble intensity [19]. In addition, in [30], the authors deduce the equivalency between Poisson model and Gaussian model. Therefore, both theories indicate that these two noises have similar statistical properties and can be unified into a whole framework.

\section{Measure Similarity}

The formula of bilateral filter is

$$
B\left(y_{i j}\right)=\frac{1}{k\left(y_{i j}\right)} \iint_{-\infty}^{\infty} y_{s t} c((s, t),(i, j)) s\left(y_{s t}, y_{i j}\right) d s d t
$$

where $(s, t)$ and $(i, j)$ are two pixels of sinogram. Here, sinogram is the observations of projection data, that is, the noisy projection data of LDCT. $y_{s t}$ and $y_{i j}$ are sinogram values of $(s, t)$ and $(i, j)$, respectively. $k\left(y_{i j}\right)$ is a normalized constant for two weighs and is defined as

$$
k\left(y_{i j}\right)=\iint_{-\infty}^{\infty} c((s, t),(i, j)) s\left(y_{s t}, y_{i j}\right) d s d t
$$

where $c((s, t),(i, j))$ and $s\left(y_{s t}, y_{i j}\right)$ are measures of the spatial and range similarity between the center pixel $y_{i j}$ and its neighbor $y_{s t}$, respectively. Usually, these two measures are defined as two Gaussian Kernel functions

$$
\begin{gathered}
c((s, t),(i, j))=e^{(-1 / 2)\left(\|(s, t)-(i, j)\| / \sigma_{d}\right)^{2}}, \\
s\left(y_{s t}, y_{i j}\right)=e^{(-1 / 2)\left(\left\|y_{s t}-y_{i j}\right\| / \sigma_{r}\right)^{2}} .
\end{gathered}
$$

Since the $(i, j)$ value filtered by BF is the weighted average of nearby points weighted by product of spatial distance and gray level difference, it was named by bilateral filter (BF) to distinguish the general filter weighted only by spatial distance.

From (3.1)-(3.4), we can conclude that a pair of pixels $y_{s t}, y_{i j}$ with both small spatial distance and small sinogram value difference have high similarity and large-weighed coefficients. It is plausible in slightly noisy projection data. For sinograms with serious noise and non-stationary artifacts, it is unreal! That is, polluted sinogram values lead to incorrect similarity measurement in the range filter of the bilateral filter. Thus, finding a measure of similarity, which can measure similarity correctly in noise and non-stationary artifacts, is a key problem in denoising using BF.

\subsection{Gaussian Filter}

Gaussian filter is defined as

$$
G\left(y_{i j}\right)=\frac{1}{\iint_{-\infty}^{\infty} e^{(-1 / 2)\left(\left\|y_{s t}-y_{i j}\right\| / \sigma\right)^{2}} d s d t} \iint_{-\infty}^{\infty} y_{s t} e^{-1 / 2\left(\left\|y_{s t}-y_{i j}\right\| / \sigma\right)^{2}} d s d t
$$


Since $y_{s t} \sim N\left(\mu_{i j}, \sigma_{i j}^{2}\right)$ for $s=-\infty, \ldots, \infty$ and $t=-\infty, \ldots, \infty$, noisy sinogram value $Y_{i j} \sim$ $N\left(\mu_{i j}, \sigma_{i j}^{2}\right)$ and the Gaussian-dependent noise $(\mathrm{GWN}) N_{i j} \sim N\left(0, \sigma_{i j}^{2}\right)$, the distribution of the pixel $(i, j)$ filtered by the low-passed filter defined in (3.5) is

$$
\begin{aligned}
Y_{i j} & +e^{1 /\left(-2 \sigma^{2}\right)}\left(Y_{i-1, j}+Y_{i, j-1}+Y_{i+1, j}+Y_{i, j+1}+\cdots\right) \\
& \sim N\left(\mu_{i j}, \frac{1+\left(4 e^{1 /\left(-2 \sigma^{2}\right)}\right)^{2}+\left(4 e^{2 /\left(-2 \sigma^{2}\right)}\right)^{2}+\cdots}{\left(1+\left(4 e^{1 /\left(-2 \sigma^{2}\right)}\right)+\left(4 e^{2 /\left(-2 \sigma^{2}\right)}\right)+\cdots\right)^{2}} \sigma_{i j}^{2}\right) \\
& \sim N\left(\mu_{i j}, \frac{1+\left(4 e^{(-1) / \sigma^{2}}\right) /\left(1-e^{(-1) / \sigma^{2}}\right)}{\left(1+\left(4 e^{(-1) / 2 \sigma^{2}}\right) /\left(1-e^{(-1) / 2 \sigma^{2}}\right)\right)^{2}} \sigma_{i j}^{2}\right) .
\end{aligned}
$$

Thus,

$$
G\left(Y_{i j}\right) \sim N\left(\mu_{i j}, \frac{\left(e^{1 / \sigma^{2}}+3\right)\left(e^{1 / 2 \sigma^{2}}-1\right)^{2}}{\left(e^{1 / \sigma^{2}}-1\right)\left(e^{1 / 2 \sigma^{2}}+3\right)^{2}} \sigma_{i j}^{2}\right) .
$$

For example, in image denoising, generally, $\sigma$ is set to 2; thus,

$$
G\left(Y_{i j}\right) \sim N\left(\mu_{i j}, 0.0157 \sigma_{i j}^{2}\right)
$$

From the above equation, the variance of the smoothed sinogram value becomes very small (smaller than original variance 0.0157 times). It means that the Gaussian filter makes smoothed sinogram value closer to real projection data than the noisy sinogram value. Since most of non-stationary artifacts in image space are the high-light points in noisy sinogram, most of non-stationary artifacts can be suppressed by Gaussian filter.

In the same way, the distribution of the median in an $s \times s$ window centered at the pixel $(i, j)$ is

$$
\operatorname{median}\left(Y_{i j}\right) \sim N\left(\mu_{i j}, \frac{\sigma_{i j}^{2}}{s \times s}\right)
$$

Just as the above discussion, if the median filter has similar estimate precision to Gaussian filter in image denoising, $s$ should at least be 8 , which is estimated by $\sqrt{1 / 0.0157}=$ $\sqrt{63.6943} \approx 8$. However, so large window of median filter will delete some real lines in sinogram, which will lead to many artifacts in denoising sinogram.

\subsection{Similarity Discussion}

From the second equation of (3.4), the similarity between the sinogram values of two pixels $(i, j)$ and $(s, t)$ is defined as a Gaussian function of the difference to their sinogram values. Thus, large difference has small similarity, while small difference has large similarity. 
Following this conclusion, similarity discussion can be accomplished by discussing the difference for each pair of pixels of sinogram. In this subsection, we will discuss the differences by variances of three denoising schemes for BF.

Assume that $Y_{s t}$ and $Y_{i j}$ are iid Gaussian random variables corresponding to a pair of pixels with the same real gray levels, $Y_{i j} \sim N\left(\mu_{i j}, \sigma_{i j}^{2}\right), Y_{s t} \sim N\left(\mu_{i j}, \sigma_{i j}^{2}\right)$, and their difference

$$
Y_{i j}-Y_{s t} \sim N\left(\mu_{i j}, 2 \sigma_{i j}^{2}\right)
$$

In the same way, since median $\left(Y_{i j}\right) \sim N\left(\mu_{i j}, \sigma_{i j}^{2} / s \times s\right)$, we can conclude that

$$
\operatorname{median}\left(Y_{i j}\right)-Y_{s t} \sim N\left(\mu_{i j}, \frac{1+s^{2}}{s^{2}} \sigma_{i j}^{2}\right)
$$

$$
\begin{aligned}
& \text { Since } G\left(Y_{i j}\right) \sim N\left(\mu_{i j},\left(\left(\left(e^{1 / \sigma^{2}}+3\right)\left(e^{1 / 2 \sigma^{2}}-1\right)^{2}\right) /\left(e^{1 / \sigma^{2}}-1\right)\left(e^{1 / 2 \sigma^{2}}+3\right)^{2}\right) \sigma_{i j}^{2}\right) \text {, thus } \\
& G\left(Y_{i j}\right)-G\left(Y_{s t}\right) \sim N\left(\mu_{i j}, 2 \frac{\left(e^{1 / \sigma^{2}}+3\right)\left(e^{1 / 2 \sigma^{2}}-1\right)^{2}}{\left(e^{1 / \sigma^{2}}-1\right)\left(e^{1 / 2 \sigma^{2}}+3\right)^{2}} \sigma_{i j}^{2}\right)
\end{aligned}
$$

Just as discussed in the last subsection, if we set $\sigma$ to 2 ,

$$
G\left(Y_{i j}\right)-G\left(Y_{s t}\right) \sim N\left(\mu_{i j}, 0.0314 \sigma_{i j}^{2}\right) .
$$

It is obvious that the variance of the first scheme is the biggest in all three schemes, while the variance of the last scheme is the smallest in all three schemes. Since $s \geq 3$, we have

$$
2>\frac{s^{2}+1}{s^{2}}>1>0.0314
$$

The first scheme corresponds to the bilateral, which measures difference by the sinogram values of $(i, j)$ and $(s, t)$ directly. The second scheme corresponds to the mean bilateral proposed in [41] whose similarity is measured between the median of the center pixel $(i, j)$ and the sinogram value of its neighbor $(s, t)$. The third scheme corresponds to the scheme of measuring the difference on the Gaussian filtered sinogram value.

It is well-known that smallest variance corresponds to the best estimate of real projection data value. According to this rule, our proposed method can provide the best estimate of real projection data value. Thus, BFWGFS can reduce both the influence of noises and non-stationary artifacts.

\section{The Algorithm}

Just as the above discussion, satisfied denoising results can be got by weighed range filter on Gaussian filtered sinogram. The steps of the algorithm are as follows: 
(1) compute the Gaussian filtered sinogram value $G\left(y_{i j}\right)$ for all sinogram pixels using (3.5),

(2) give $\sigma_{d}$ and $\sigma_{r}$,

(3) for each of pixel,

(i) compute $c((s, t),(i, j))$ using the first equation of (3.4) and $s\left(G\left(y_{s t}\right), G\left(y_{i j}\right)\right)$ using

$$
s\left(G\left(y_{s t}\right), G\left(y_{i j}\right)\right)=e^{(-1 / 2)\left(\left\|G\left(y_{s t}\right)-G\left(y_{i}\right)\right\| / \sigma_{r}\right)^{2}},
$$

(ii) compute $k\left(y_{i j}\right)$ using

$$
k\left(y_{i j}\right)=\iint_{-\infty}^{\infty} c((s, t),(i, j)) s\left(G\left(y_{s t}\right), G\left(y_{i j}\right)\right) d s d t
$$

(iii) compute $G B\left(y_{i j}\right)$ using

$$
G B\left(y_{i j}\right)=\frac{1}{k\left(y_{i j}\right)} \iint_{-\infty}^{\infty} y_{s t} c((s, t),(i, j)) s\left(G\left(y_{s t}\right), G\left(y_{i j}\right)\right) d s d t
$$

(4) repeat step 3 until all sinogram pixels have been proceeded.

\section{Experiments and Discussion}

The main objective for smoothing L-CT images is to delete the noise and non-stationary artifacts while to preserve anatomy details for the images. Thus, the image visual quality can be improved, and the denoised image can help doctors make correct medical diagnosis more easily.

\subsection{Data}

Four groups of CT images with different doses were scanned from a 16 multidetector row CT unit (Somatom Sensation 16; Siemens Medical Solutions) using $120 \mathrm{kVp}$ and $5 \mathrm{~mm}$ slice thickness: a 58-year-old man, two groups of 62-year-old women with different reduced dose, and a 60 -year-old man. Other remaining scanning parameters are gantry rotation time, 0.5 second; detector configuration (number of detector rows section thickness), $16 \times 1.5 \mathrm{~mm}$; table feed per gantry rotation, $24 \mathrm{~mm}$; pitch, $1: 1$; reconstruction method, filtered back projection (FBP) algorithm with the soft-tissue convolution kernel "B30f." Different CT doses were controlled by using two different fixed tube currents $30 \mathrm{mAs}$ and $150 \mathrm{mAs}$ (60 mA or 300 $\mathrm{mAs}$ ) for LDCT and standard-dose CT (SDCT) protocols, resp.. The CT dose index volume (CTDIvol) for LDCT images and SDCT images are in positive linear correlation to the tube current and is calculated to be approximately ranged between $15.32 \mathrm{mGy}$ and $3.16 \mathrm{mGy}$ [29]. For additional visually illustration, we also put two groups of abdominal CT images of a same woman with $60 \mathrm{mAs}$, and two groups of shoulder CT images with low dose $35 \mathrm{mAs}$ and standard dose $135 \mathrm{mAs}$ (see Figure 2). 


\subsection{Compared Methods}

Bilateral filter $(B F)$ is introduced at the beginning of Section 3. The main motivation for BF is that the noisy image should be weighted not only by the position distance (spatial filter) but also by the difference of sinogram values (range filter) [35]. The parameters of BF are Gaussian Kernel for spatial filter $\sigma_{s}=1.8$, Gaussian Kernel for range filter $\sigma_{r}=20 / 3$, and iteration time is 3 .

Context is a term imported from image coding. The context of a pixel $x_{i j}$ is always defined as a vector used for describing the relationship between this pixels and other image pixels. In this paper, in order to suppress the influence of noises, the context is defined as

$$
\widehat{y}_{i j}=\frac{1}{9} \sum_{s=i-1}^{s=i+1} \sum_{t=j-1}^{t=j+1} y_{s t}
$$

The context filter estimates real sinogram values from the points with similar context value. In this paper, the threshold value for similar context is 10 , that is,

$$
\begin{gathered}
x_{i j} \text { and } x_{s t} \text { are similar points if }\left|\widehat{y}_{i j}-\widehat{y}_{s t}\right| \leq 10, \\
x_{i j} \text { and } x_{s t} \text { are not similar points otherwise, }
\end{gathered}
$$

where $\widehat{y}_{i j}$ is defined on (5.1). Although context filter can provide more samples for real value estimate, it will produce some artifacts for losing the spatial relationship of sinogram.

Median bilateral filter (MBF) replaces the center pixel with the median of an $s \times s$ window [41]. However, just as analysis in Section 3, only replacing the center pixel also cannot obtain satisfied denoising results. Here, when $s$ set to 5 has the best performance, $\sigma_{r}=20 / 3$ and $\sigma_{s}=1.8$.

Multiresolution bilateral filter (MRBF) filtering LL subband uses BF while smoothing wavelet subbands uses SURE shrinkage [38]. The wavelet used in the experiment is 1-level symlets with support 4 . The noisy variance $\widehat{\sigma}_{N}$ is estimated using median of $\mathrm{HH}$ band of the wavelet [45] and $\sigma_{r}=3 \widehat{\sigma}_{N}$ and $\sigma_{s}=2$. Although authors report that MRBF can obtain good denoising results, it also leads to blur some important details.

Weighted intensity averaging over large-scale neighborhoods (WIA-LNs) is a state-of-the-art method for sinogram reconstruction [29]. The motivation for WIA-LN is that the two pixels of the same organ or tissue should have surrounding patches with higher similarities than the two pixels of different organs or tissues. Thus, the real sinogram value of $f_{i}$ can be estimated as

$$
\widehat{f}_{i}=\sum_{j \in N_{i}} \frac{\omega_{i j} f_{i}}{\sum_{j \in N_{i}} \omega_{i j}}
$$

where

$$
\omega_{i j}=\exp \left(-\frac{\left\|n_{i}-n_{j}\right\|_{2, \alpha}^{2}}{\beta\left|n_{i}\right|}\right) .
$$


Here, $f_{i}$ denotes the intensities of the neighboring pixels in the search neighborhood $N_{i}$ centered at pixel index $i$. The weight of WIA-LN is built by using a similarity criterion between the two comparing patches $n_{i}$ and $n_{j}$. This similarity metrics is calculated using (5.4), in which $\alpha$ denotes the two-dimensional standard deviation of Gaussian kernel. $\left|n_{i}\right|$ is the total pixel number in patch $n_{i} . \beta$ is a superparameter. In this paper, $\beta$ is set to be 0.8 , and the sizes $n_{i}$ are set to $11 \times 11$. Although better vision and quantitative performance are reported, the authors also indicate that WIA-LN cannot handle noise and non-stationary artifacts simultaneously (see Figure $3(\mathrm{~g})$ ).

Proposed method (BFWGFS) replaces all sinogram values used in range filter of BF by the Gaussian filtered sinogram values. Just as discussed in Section 3, smoothed sinogram values can reduce the influence of both noise and non-stationary artifacts greatly, and BFWGFS can provide good visual results and preserve more anatomy details. The parameters are $\sigma_{r}=$ $20 / 3, \sigma_{s}=1.8$, iteration number is set to 1 , and Gaussian smoothed kernel is set to 1.8.

\subsection{Visual Comparison}

Three groups of SDCT images, LDCT images, and the processed LDCT images for the clinical abdominal examinations are shown in Figures 1-3. The parameters for compared methods have been given in the last subsection. In Figure 1, the original and processed abdominal CT images of a 58-year-old man are illustrated. Figures 1(a) and 1(b) are one SDCT image and one LDCT image acquired at tube current time product $150 \mathrm{mAs}$ and $30 \mathrm{mAs}$, respectively. Figures 1(c), 1(d), 1(e), 1(f), 1(g), and 1(h) show BF, context, MBF, MRBF, WIA-LN, and proposed method processed LDCT images, respectively. Figure 2 illustrates the original and processed abdominal CT images of a 62-year-old woman. Figure 2(a) is one SDCT image acquired at tube current time product $150 \mathrm{mAs}$. Figures 2(b) and 2(c) are two LDCT images acquired at reduced tube current time products $60 \mathrm{mAs}$ and $30 \mathrm{mAs}$, respectively. Figures 2(d), 2(e), 2(f), 2(j), 2(k), 2(l), 2(g), 2(h), 2(i), 2(m), 2(n), and 2(o) illustrate the two groups of processed LDCT images of Figures 2(b) and 2(c) by using compared methods. Figure 3 illustrates the original and processed images for one shoulder scan of a 60-yearold man, from which we found that WIA-LN tends to smooth both the streak artifacts and informative human tissues, while proposed method can reduce the noise and artifacts with preservation of anatomy details.

Comparing all the original SDCT images and LDCT images in Figures 1-3, we found that the LDCT images were severely degraded by noise and streak artifacts. In Figures 1(c)$1(\mathrm{f})$, just as the discussion in Section 3, there are so many noises left in processed images using BF, context, MBF, and MRBF. WIA-LN shown in Figure $1(\mathrm{~g})$ also makes some obvious artifacts, while we can observe better noise/artifacts suppression and edge preservation for proposed methods in Figure 1(h). Both WIA-LN and proposed method have good performance in noises. Especially, compared to corresponding original SDCT images, the fine features representing the intrahepatic bile duct dilatation and the hepatic cyst (pointed by the white circles in the images of Figures 1 and 2, resp.) were well restored by using WIA-LN and proposed method. The fine anatomical/pathological features (the exemplary structures pointed by circles in Figures 1 and 2) can be well preserved compared to the original SDCT images (Figures 1(a) and 2(a)) under-standard dose conditions. In Figures 3(g) and 3(h), it indicates that although WIA-LN cannot handle noises and artifacts simultaneously, proposed method can obtain satisfied results in this complex situation. Especially, proposed method not only can suppress noises and artifacts in original LDCT image (Figure 3(a)) but also 


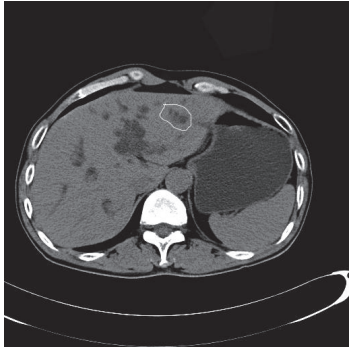

(a)

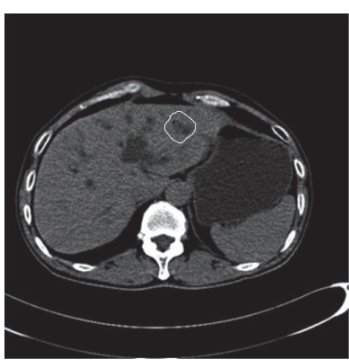

(d)

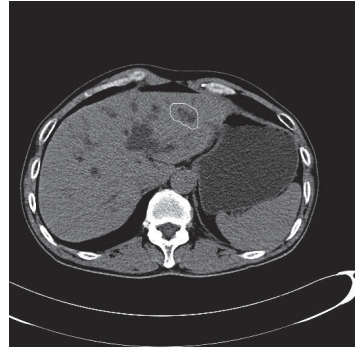

(b)

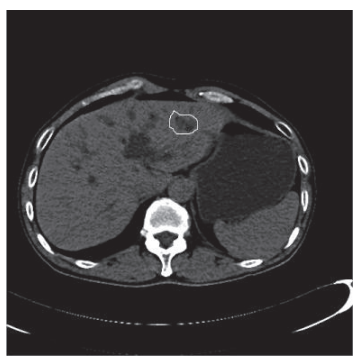

(e)

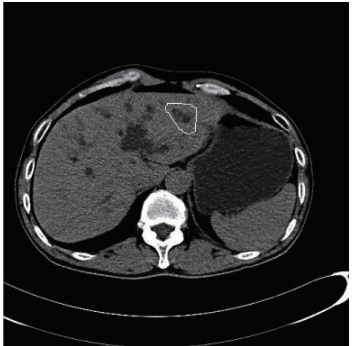

(c)

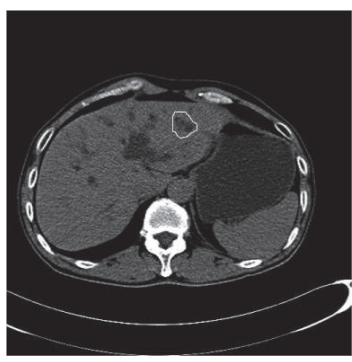

(f)

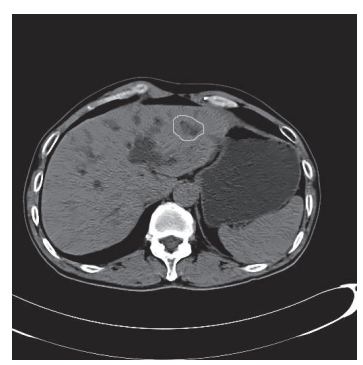

(g)

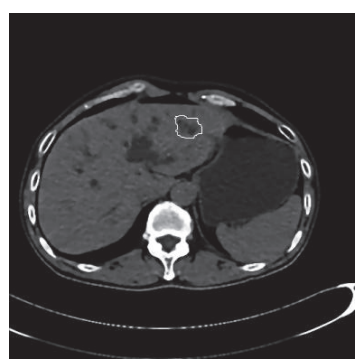

(h)

Figure 1: Abdominal CT images of a 58-year-old man. (a) Original SDCT image with tube current time product 150 mAs. (b) Original LDCT image with tube current time product 30 mAs. (c, d, e, f) BF, context, MBF, and MRBF processed LDCT images, respectively. (g) WIA-LN processed LDCT image. (h) Proposed method (BFWGFS) processed LDCT image. Note the obvious improvement of noise suppression and preservation of the intrahepatic bile duct dilatation (white circles) for the WIA-LN and proposed method processed LDCT images $(\mathrm{g}, \mathrm{h})$ compared to the original LDCT image in $(\mathrm{b})$.

can preserve tiny anatomy details of subscapular arteries indicated by the white circles in Figure 3(h) compared to the original SDCT image (Figure 3(b)).

\section{Conclusions}

In this paper, in order to improve the performance of LDCT imaging, we propose a new method, named bilateral filter weighted by Gaussian filtered sinogram (BFWGFS) which replaces the sinogram values of range filter of $\mathrm{BF}$ to the Gaussian filtered sinogram values. Since carefully chosen parameters of Gaussian filter can reduce the influence both of noises and non-stationary artifacts greatly, BFWGFS can provide a more reliable estimate sinogram values for the range filter to improve the performance of classical BF in noises. Restoration 


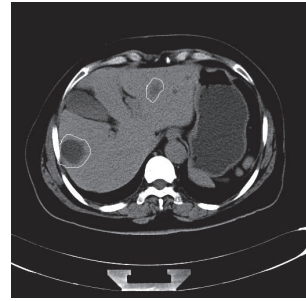

(a)

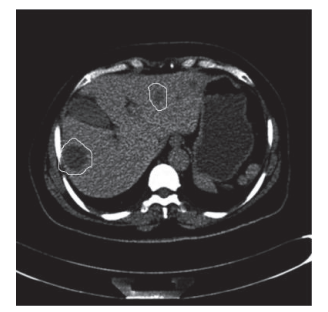

(e)

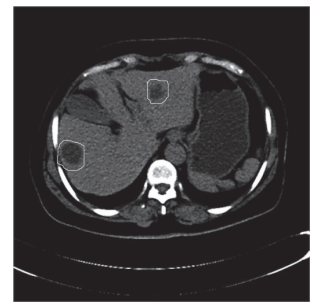

(i)

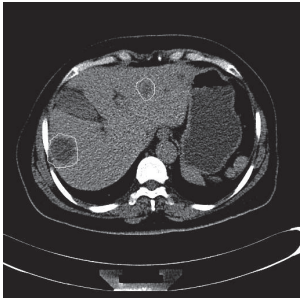

(b)

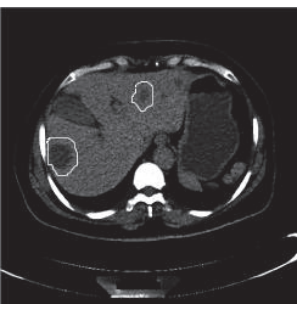

(f)

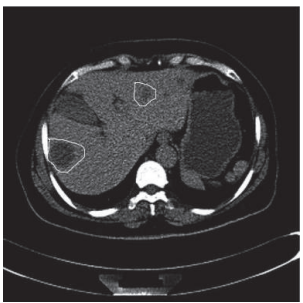

(j)

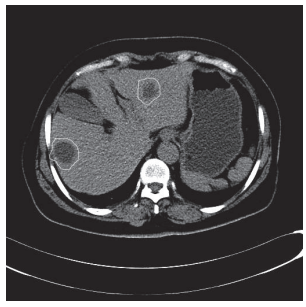

(c)

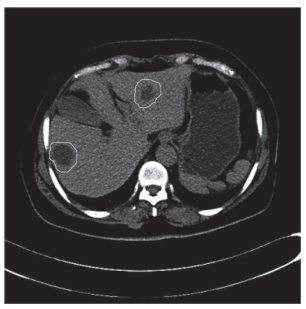

(g)

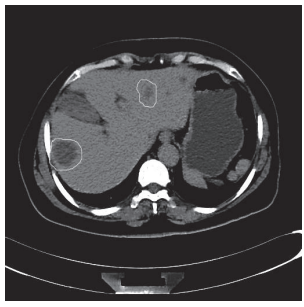

$(\mathrm{k})$

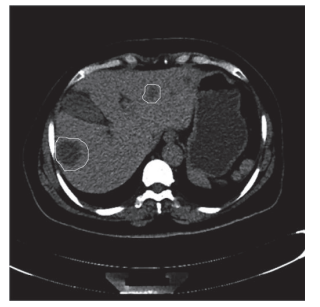

(d)

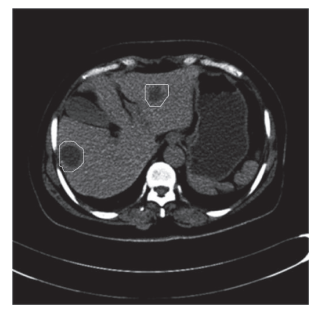

(h)

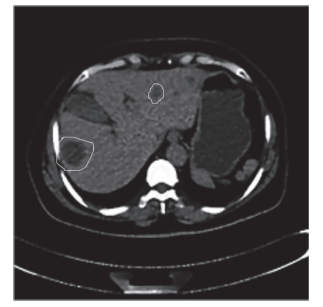

(1)

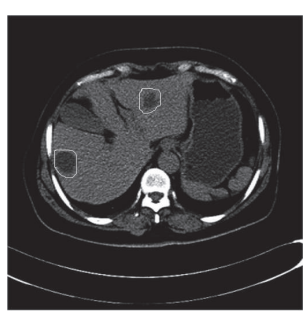

(m)

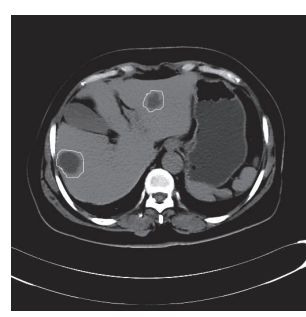

(n)

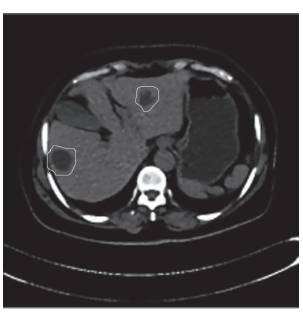

(o)

Figure 2: Abdominal CT images of a 62-year-old woman. (a) Original SDCT image with tube current time product $150 \mathrm{mAs}$. (b) Original LDCT image with tube current time product $60 \mathrm{mAs}$. (c) Original LDCT image with tube current time product $30 \mathrm{mAs}$. (d, e, f) LDCT images (60 mAs) processed by BF, context, and MBF, respectively. $(\mathrm{g}, \mathrm{h}, \mathrm{i}) \mathrm{LDCT}$ images $(30 \mathrm{mAs})$ processed by $\mathrm{BF}$, context, and MBF, respectively. ( $\mathrm{j}$, $\mathrm{k}, \mathrm{l})$ LDCT images (60 mAs) processed by MRBF, WIA-LN and proposed method, respectively. $(\mathrm{m}, \mathrm{n}, \mathrm{o})$ LDCT images $(30 \mathrm{mAs})$ processed by MRBF, WIA-LN, and proposed method, respectively. Compared to the original LDCT images in (b) and (c), the improvement of preservation of the hepatic cyst (white circles) for the WIA-LN, and proposed method in processed LDCT image (k), (1), and (n), (o) can be observed.

results for three real sinograms show that proposed method with suitable parameters can obtain satisfied results even in both the noises and artifacts situation.

\section{Acknowledgments}

This paper is supported by the National Natural Science Foundation of China (no. 60873102), Major State Basic Research Development Program (no. 2010CB732501), and Open Foundation 


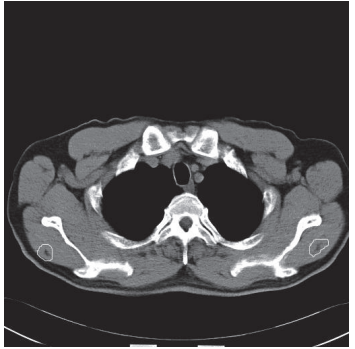

(a)

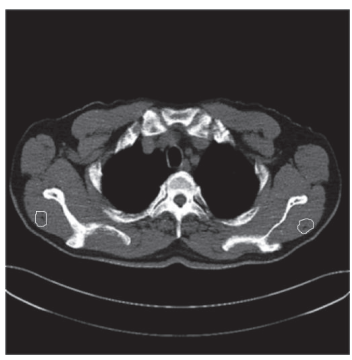

(d)

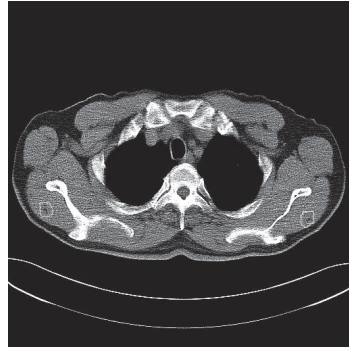

(b)

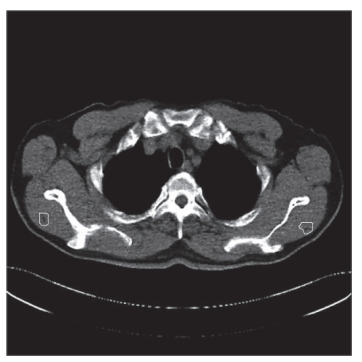

(e)

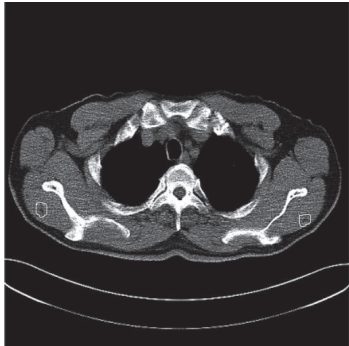

(c)

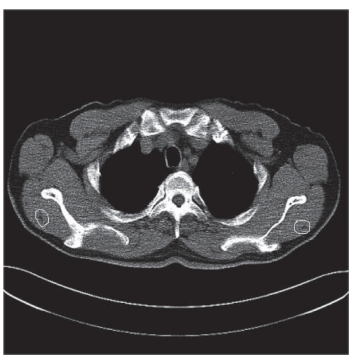

(f)

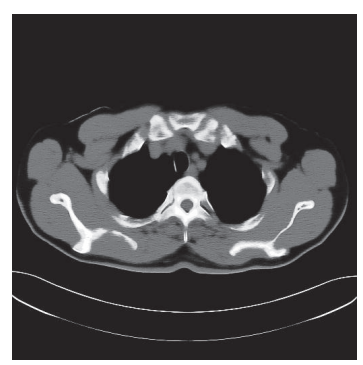

(g)

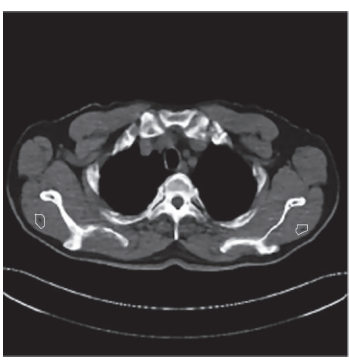

(h)

Figure 3: Shoulder CT images of a 60-year-old man. (a) Original SDCT image acquired at a standard tube current-time product $135 \mathrm{mAs}$. (b) Original LDCT image acquired at a reduced tube current-time product $35 \mathrm{mAs}$. (c, f, g, h) LDCT images processed by BF, context, MBF, and MRBF, respectively. (d) LDCT image processed by WIA-LN. Note that both the streak artifacts and informative human tissues tend to be smoothed. (e) LDCT image processed by the proposed method. Compared to the original LDCT images in (a), the obvious improvement of noise suppression in the improvement of preservation of the subscapular arteries (white circles) for the proposed method can be observed.

of Visual Computing and Virtual Reality Key Laboratory of Sichuan Province (no. J2010N03). This work was supported by a Grant from the National High Technology Research and Development Program of China (no. 2009AA12Z140).

\section{References}

[1] D. J. Brenner and E. J. Hall, "Computed tomography-an increasing source of radiation exposure," New England Journal of Medicine, vol. 357, no. 22, pp. 2277-2284, 2007.

[2] J. Hansen and A. G. Jurik, "Survival and radiation risk in patients obtaining more than six CT examinations during one year," Acta Oncologica, vol. 48, no. 2, pp. 302-307, 2009. 
[3] H. J. Brisse, J. Brenot, N. Pierrat et al., "The relevance of image quality indices for dose optimization in abdominal multi-detector row CT in children: experimental assessment with pediatric phantoms," Physics in Medicine and Biology, vol. 54, no. 7, pp. 1871-1892, 2009.

[4] L. Yu, "Radiation dose reduction in computed tomography: techniques and future perspective," Imaging in Medicine, vol. 1, no. 1, pp. 65-84, 2009.

[5] J. Weidemann, G. Stamm, M. Galanski, and M. Keberle, "Comparison of the image quality of various fixed and dose modulated protocols for soft tissue neck CT on a GE Lightspeed scanner," European Journal of Radiology, vol. 69, no. 3, pp. 473-477, 2009.

[6] W. Qi, J. Li, and X. Du, "Method for automatic tube current selection for obtaining a consistent image quality and dose optimization in a cardiac multidetector CT," Korean Journal of Radiology, vol. 10, no. 6, pp. 568-574, 2009.

[7] A. Kuettner, B. Gehann, J. Spolnik et al., "Strategies for dose-optimized imaging in pediatric cardiac dual source CT," Fortschr. Röntgenstr, vol. 181, no. 4, pp. 339-348, 2009.

[8] P. Kropil, R. S. Lanzman, C. Walther et al., "Dose reduction and image quality in MDCT of the upper abdomen: potential of an adaptive post-processing filter," Fortschr. Röntgenstr, vol. 182, no. 3, pp. 284 253, 2009.

[9] M. K. Kalra, M. M. Maher, M. A. Blake et al., "Detection and characterization of lesions on lowradiation-dose abdominal CT images postprocessed with noise reduction filters," Radiology, vol. 232, no. 3, pp. 791-797, 2004.

[10] H. B. Lu, X. Li, L. H. Li et al., "Adaptive noise reduction toward low-dose computed tomography," in Proceedings of the Medical Imaging 2003: Physics of Medical Imaging, vol. 5030, parts 1 and 2, pp. 759-766, San Diego, Calif, USA, February 2003.

[11] M. K. Kalra, C. Wittram, M. M. Maher et al., "Can noise reduction filters improve low-radiation-dose chest CT images? Pilot study," Radiology, vol. 228, no. 1, pp. 257-264, 2003.

[12] M. K. Kalra, M. M. Maher, D. V. Sahani et al., "Low-dose CT of the abdomen: evaluation of image improvement with use of noise reduction filters pilot study," Radiology, vol. 228, no. 1, pp. 251-256, 2003.

[13] J. C. Giraldo, Z. S. Kelm, L. S. Guimaraes et al., "Comparative study of two image space noise reduction methods for computed tomography: bilateral filter and nonlocal means," in Proceedings of the 31st Annual International Conference of the IEEE Engineering in Medicine and Biology Society: Engineering the Future of Biomedicine (EMBC '09), pp. 3529-3532, Minneapolis, Minn, USA, September 2009.

[14] A. Manduca, L. Yu, J. D. Trzasko et al., "Projection space denoising with bilateral filtering and CT noise modeling for dose reduction in CT," Medical Physics, vol. 36, no. 11, pp. 4911-4919, 2009.

[15] N. Mail, D. J. Moseley, J. H. Siewerdsen, and D. A. Jaffray, "The influence of bowtie filtration on cone-beam CT image quality," Medical Physics, vol. 36, no. 1, pp. 22-32, 2009.

[16] M. Kachelrie, O. Watzke, and W. A. Kalender, "Generalized multi-dimensional adaptive filtering for conventional and spiral single-slice, multi-slice, and cone-beam CT," Medical Physics, vol. 28, no. 4, pp. 475-490, 2001.

[17] G. F. Rust, V. Aurich, and M. Reiser, "Noise/dose reduction and image improvements in screening virtual colonoscopy with tube currents of $20 \mathrm{mAs}$ with nonlinear Gaussian filter chains," in Medical Imaging 2002: Physiology and Function from Multidimensional Images, vol. 4683 of Proceedings of SPIE, pp. 186-197, San Diego, Calif, USA, February 2002.

[18] Z. Liao, S. Hu, and W. Chen, "Determining neighborhoods of image pixels automatically for adaptive image denoising using nonlinear time series analysis," Mathematical Problems in Engineering, vol. 2010, Article ID 914564, 14 pages, 2010.

[19] H. Lu, I. T. Hsiao, X. Li, and Z. G. Liang, "Noise properties of low-dose CT projections and noise treatment by scale transformations," in Proceedings of the IEEE Nuclear Science Symposium Conference Record, vol. 1-4, pp. 1662-1666, November 2001.

[20] J. Xu and B. M. W. Tsui, "Electronic noise modeling in statistical iterative reconstruction," IEEE Transactions on Image Processing, vol. 18, no. 6, pp. 1228-1238, 2009.

[21] I. A. Elbakri and J. A. Fessler, "Statistical image reconstruction for polyenergetic X-ray computed tomography," IEEE Transactions on Medical Imaging, vol. 21, no. 2, pp. 89-99, 2002.

[22] P. J. la Rivire and D. M. Billmire, "Reduction of noise-induced streak artifacts in X-ray computed tomography through spline-based penalized-likelihood sinogram smoothing," IEEE Transactions on Medical Imaging, vol. 24, no. 1, pp. 105-111, 2005.

[23] P. J. la Rivire, "Penalized-likelihood sinogram smoothing for low-dose CT," Medical Physics, vol. 32, no. 6, pp. 1676-1683, 2005. 
[24] J. Wang, H. Lu, J. Wen, and Z. G. Liang, "Multiscale penalized weighted least-squares sinogram restoration for low-dose X-ray computed tomography," IEEE Transactions on Biomedical Engineering, vol. 55, no. 3, pp. 1022-1031, 2008.

[25] P. Forthmann, T. Kohler, P. G. Begemann, and M. Defrise, "Penalized maximum-likelihood sinogram restoration for dual focal spot computed tomography," Physics in Medicine and Biology, vol. 52, no. 15, pp. 4513-4523, 2007.

[26] J. Wang, T. Li, H. Lu, and Z. G. Liang, “Penalized weighted least-squares approach to sinogram noise reduction and image reconstruction for low-dose X-ray computed tomography," IEEE Transactions on Medical Imaging, vol. 25, no. 10, pp. 1272-1283, 2006.

[27] Z. Liao, S. Hu, M. Li, and W. Chen, "Noise estimation for single-slice sinogram of low-dose X-ray computed tomography using homogenous patch," Mathematical Problems in Engineering, vol. 2012, Article ID 696212, 16 pages, 2012.

[28] H. B. Lu, X. Li, I. T. Hsiao, and Z. G. Liang, "Analytical noise treatment for low-dose CT projection data by penalized weighted least-square smoothing in the K-L domain," in Proceedings of the Medical Imaging 2002: Physics of Medical Imaging, vol. 4682, pp. 146-152, May 2002.

[29] C. Yang, C. Wufan, Y. Xindao et al., "Improving low-dose abdominal CT images by weighted intensity averaging over large-scale neighborhoods," European Journal of Radiology, vol. 80, no. 2, pp. e42-e49, 2011.

[30] T. Li, X. Li, J. Wang et al., “Nonlinear sinogram smoothing for low-dose X-ray CT,” IEEE Transactions on Nuclear Science, vol. 51, no. 5, pp. 2505-2513, 2004.

[31] S. Hu, Z. Liao, D. Sun, and W. Chen, "A numerical method for preserving curve edges in nonlinear anisotropic smoothing," Mathematical Problems in Engineering, vol. 2011, Article ID 186507, 14 pages, 2011.

[32] M. Li and W. Zhao, "Visiting power laws in cyber-physical networking systems," Mathematical Problems in Engineering, vol. 2012, Article ID 302786, 13 pages, 2012.

[33] M. Li, C. Cattani, and S. Y. Chen, "Viewing sea level by a one-dimensional random function with long memory," Mathematical Problems in Engineering, vol. 2011, Article ID 654284, 13 pages, 2011.

[34] M. Li, "Fractal time series: a tutorial review," Mathematical Problems in Engineering, vol. 2010, Article ID 157264, 26 pages, 2010.

[35] C. Tomasi and R. Manduchi, "Bilateral filtering for gray and color images," in Proceedings of the IEEE International Conference on Computer Vision, pp. 836-846, Bombay, India, January 1998.

[36] D. Barash, "A fundamental relationship between bilateral filtering, adaptive smoothing, and the nonlinear diffusion equation," IEEE Transactions on Pattern Analysis and Machine Intelligence, vol. 24, no. 6, pp. 844-847, 2002.

[37] A. Wong, "Adaptive bilateral filtering of image signals using local phase characteristics," Signal Processing, vol. 88, no. 6, pp. 1615-1619, 2008.

[38] Z. Ming and G. Bahadir, "Multiresolution bilateral filtering for image denoising," IEEE Transactions on Image Processing, vol. 17, no. 12, pp. 2324-2333, 2008.

[39] H. Yu, L. Zhao, and H. Wang, "Image denoising using trivariate shrinkage filter in the wavelet domain and joint bilateral filter in the spatial domain," IEEE Transactions on Image Processing, vol. 18, no. 10, pp. 2364-2369, 2009.

[40] R. van Boomgaard and J. van de Weijer, "On the equivalence of local-mode finding,robust estimation and mean-shift analysis as used in early vision tasks," in Proceedings of the 16th International Conference on Pattern Recognition, vol. 3, pp. 972-930, Quebec, Canada, August 2002.

[41] J. J. Francis and G. de Jager, "The bilateral median filter," Transactions of the South African Institute of Electrical Engineers, vol. 96, no. 2, pp. 106-111, 2005.

[42] K. Dabov, A. Foi, V. Katkovnik, and K. Egiazarian, “Image denoising by sparse 3D transform-domain collaborative filtering," IEEE Transactions on Image Processing, vol. 16, no. 8, pp. 2080-2095, 2007.

[43] J. Portilla, V. Strela, M. J. Wainwright, and E. P. Simoncelli, "Image denoising using scale mixtures of Gaussians in the wavelet domain," IEEE Transactions on Image Processing, vol. 12, no. 11, pp. 1338-1351, 2003.

[44] A. Buades, B. Coll, and J.-M. Morel, "Nonlocal image and movie denoising," International Journal of Computer Vision, vol. 76, no. 2, pp. 123-139, 2008, Special section: selection of papers for CVPR 2005, guest editors: C. Schmid, S. Soatto and C. Tomasi.

[45] D. L. Donoho and I. M. Johnstone, "Ideal spatial adaptation by wavelet shrinkage," Biometrika, vol. 81, no. 3, pp. 425-455, 1994. 


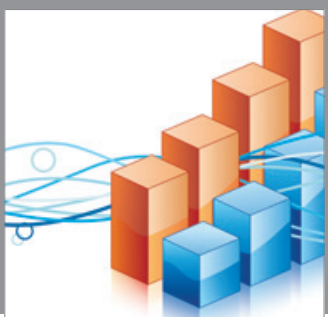

Advances in

Operations Research

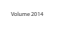

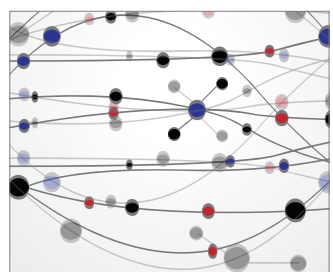

\section{The Scientific} World Journal
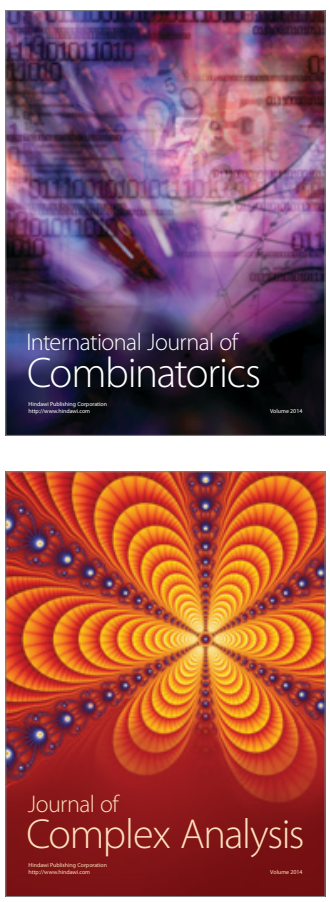

International Journal of

Mathematics and

Mathematical

Sciences
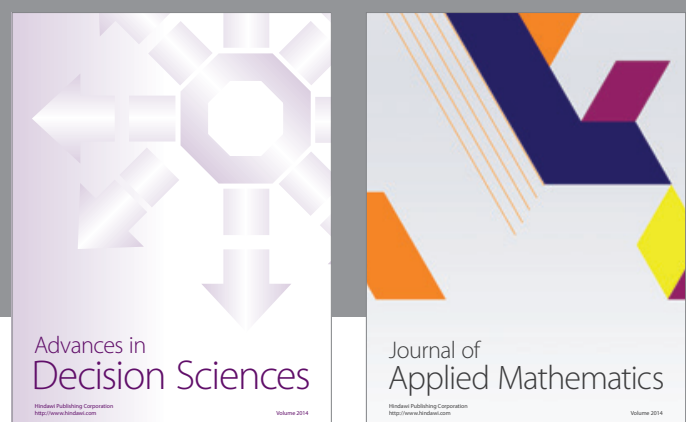

Journal of

Applied Mathematics
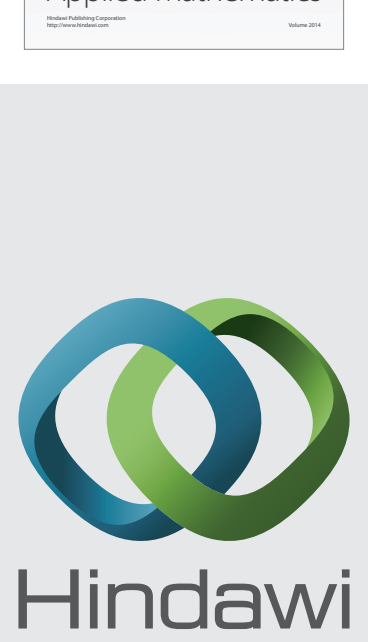

Submit your manuscripts at http://www.hindawi.com
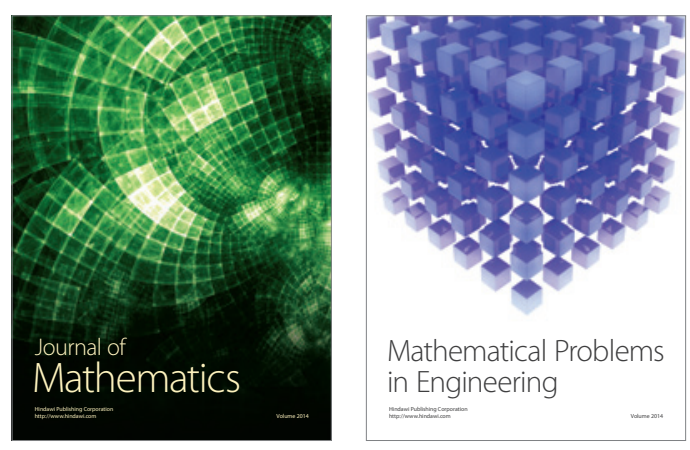

Mathematical Problems in Engineering
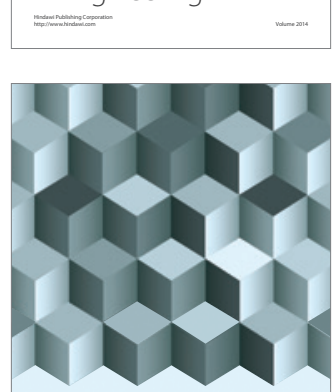

Journal of

Function Spaces
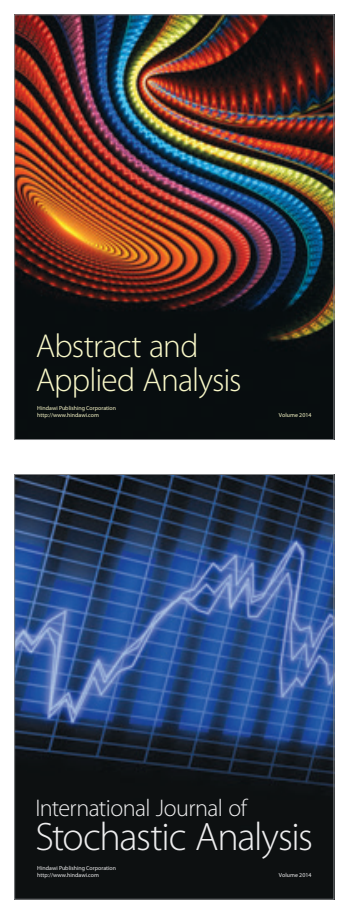

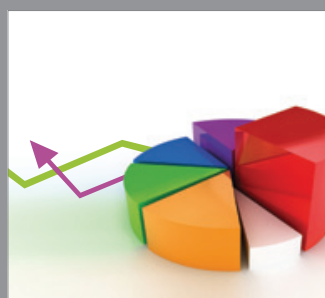

ournal of

Probability and Statistics

Promensencen
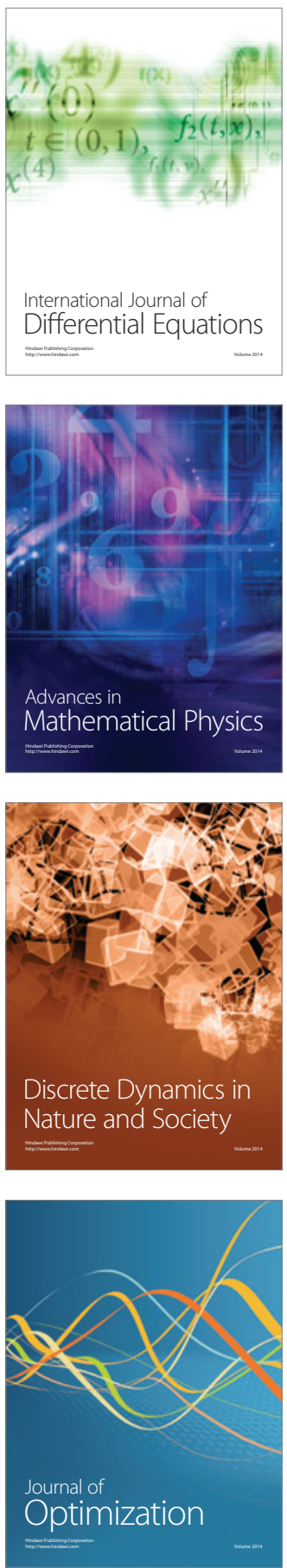